\title{
A Simulation Study on toll gate system in M/M/1 Queueing Models
}

\author{
S. Shanmugasundaram ${ }^{*}$ and S.Punitha ${ }^{* *}$ \\ * Department of Mathematics, Government Arts College, Salem - 636007. \\ ** Department of Mathematics, V.M.K.V. Engineering College, Periyaseeragapadi, Salem-636 308.
}

\begin{abstract}
In this paper, we analyze the application of Simulation in Queueing model in tollgate are discussed. We visualize the activities of each lane is discussed. The main aim of this paper is, the management can easily find which system are busy and which system is ideal and the movement of each type of vehicles in each lane is simulated by the arrival and departure time. The numerical studies for various aspects are discussed.
\end{abstract}

Keywords: Mathematical model, Quantitative model, M/M/l Queueing model, Simulation, Probability distribution.

\section{Introduction}

Queueing theory is originated in telephony with the work of Erlang [8]. His pioneering work stimulated many authors to develop a variety of queueing models incorporating various arrival patterns, various service time distributions. Queueing theory is the mathematical study of waiting lines. In this, a model is constructed means queue lengths and waiting times can be predicted [2]. Queueing is a phenomenon in a number of fields, and it has been extensively analyzed in the study of queueing theory [4].

Queueing theory can be applied the imperfect matching between the customer and service facilities is caused by one's inability to predict accurately the arrival and service time of customers. It tries to answer the questions like, the mean waiting time in the queue, the mean system response time (waiting time in the queue plus service times), mean utilization of the service facility, distribution of the number of customers in the queue, distribution of the number of customers in the system and so forth. These questions are mainly investigated in a stochastic scenario, where e.g. the inter arrival times of the customers or the service times are assumed to be random.

Simulation is the imitation of the operation of a real world process over time [3]. The study of simulation models in tollgate simulation is a significant and the powerful force for the improvement of management operations. Now a day, it has been used to increase the efficiency, quickly obtainable and adaptable and economy of service operations. In this case study, we describe and emphasis the valuable contribution of simulation and how to help the development of operations in a project. The merging of queueing model and simulation technologies has seen a remarkable growth in recent years. The fruitful reason for the importance of simulation in queueing model is that many real world problems in operations research are too complex to be given tractable mathematical formulations. In such situations, resources can be listed in a series in the hope that at least one will give an adaptable solution.

Ultimately the striking goal in simulation and queueing model is to create a way to produce high quality solutions without any mathematical structures. It first requires that a model be developed and the model represents the behaviors of the process. Simulation can be used to predict the performance of an existing or planned system and to compare alternative solutions to a particular design problem [1]. Simulation can be used to show the eventual real effects of alternative conditions and courses of action. It's also used when the real system cannot be engaged, because it may not be accessible, or it may be dangerous or unacceptable to engage, or it is being designed but not yet to build, or it may simply not exist. [5].

Simulation approach is suitable to analyze large and complex real life problems for sensitivity analysis of complex systems. It allows the decision-maker to study the interactive system variable and the effect of changes in these variables on the system performance in order to determine the desired one. Simulation can be used to 'experiment' on a model of a real situation without incurring the costs of operating on the system and its used as a pre-service test to try out new policies and decision rules for operating a system before running the risk of experimentation in the real system.

An approach of simulation-based methods have been applied to various areas related to transportation systems and its feasibilities have been reported $[6 ; 9 ; 10 ; 14 ; 11 ; 12]$. Simulation models are expensive and take a long time to develop in sometimes and it is the trial and error approach that produces different solutions in repeated runs. Each application of simulation is ad hoc to a great extent. As a part of transportation system in Salem toll gate system is expected to provide several great advantages; improvement of usability for vehicles drivers to reduce traffic jams and a solution to environmental issues. The main reason of traffic jams in the 
tollgate in each vehicle is required to stop to pay toll fees. Over viewing the tollgate system in Salem, this paper presents the simulation model which has been developed in this study, the result of simulation provides two kinds of observations. One is management can easily find which system is busy and which system is ideal. The other one is to analyze the numerical study of the tollgate to achieve more efficient performance.

We collect the data from the tollgate near Periyar University Salem, situated in Salem Bangalore route (10kms from Salem). Here we have 4 types of vehicles category like F1 (car/jeep), F2 (Light Commercial Vehicles-LCV), F3 (Truck/Bus), F4 (Multi axel vehicles-MAV). Since the goal of the study is to propose a practical solution [7] to traffic jam issues actually occurred in the toll gate, we have designed and developed a Simulation model for toll gate in Salem as a case Study [13]. Physical layout data and measured traffic data are applied in this model and proposes some solutions derived from the simulation of the model.

Now we analyses the tollgate simulation in queueing system.

\section{Arrival Distribution Tables for $F_{1}, F_{2}, F_{3}, F_{4}$}

\begin{tabular}{|c|c|c|c|c|c|}
\hline \multicolumn{6}{|c|}{ ARRIVAL DISTRIBUTION FOR $F_{1}$} \\
\hline $\begin{array}{l}\text { S. } \\
\text { No }\end{array}$ & $\begin{array}{c}\text { Inter } \\
\text { Arrival } \\
\text { Time(min) }\end{array}$ & $\begin{array}{l}\text { No. of } \\
\text { Vehicles }\end{array}$ & Prob. & $\begin{array}{l}\text { Cum. } \\
\text { Prob. }\end{array}$ & Tag Nos. \\
\hline 1 & $0-1$ & 85 & 0.425 & 0.425 & $000-424$ \\
\hline 2 & $1-2$ & 46 & 0.23 & 0.655 & $425-654$ \\
\hline 3 & $2-3$ & 27 & 0.135 & 0.79 & $655-789$ \\
\hline 4 & $3-4$ & 14 & 0.07 & 0.86 & $790-859$ \\
\hline 5 & $4-5$ & 10 & 0.05 & 0.91 & $860-909$ \\
\hline 6 & $5-6$ & 4 & 0.02 & 0.93 & $910-929$ \\
\hline 7 & $6-7$ & 5 & 0.025 & 0.955 & $930-954$ \\
\hline 8 & $7-8$ & 1 & 0.005 & 0.96 & $955-959$ \\
\hline 9 & $8-9$ & 1 & 0.005 & 0.965 & $960-964$ \\
\hline 10 & $9-10$ & 2 & 0.01 & 0.975 & $965-974$ \\
\hline 11 & $10-11$ & 0 & - & - & - \\
\hline 12 & $11-12$ & 0 & - & - & - \\
\hline 13 & $12-13$ & 1 & 0.005 & 0.98 & $975-979$ \\
\hline 14 & $13-14$ & 0 & - & - & - \\
\hline 15 & $14-15$ & 1 & 0.005 & 0.985 & $980-984$ \\
\hline 16 & $15-16$ & 0 & - & - & - \\
\hline 17 & $16-17$ & 0 & - & - & - \\
\hline 18 & $17-18$ & 1 & 0.005 & 0.99 & $985-989$ \\
\hline 19 & $18-19$ & 1 & 0.005 & 0.995 & $990-994$ \\
\hline 20 & $19-20$ & 0 & - & - & - \\
\hline 21 & $20-21$ & 0 & - & - & - \\
\hline 22 & $21-22$ & 0 & - & - & - \\
\hline 23 & $22-23$ & 0 & - & - & - \\
\hline 24 & $23-24$ & 1 & 0.005 & 1 & 995 - 999 \\
\hline 25 & $24-25$ & 0 & - & - & - \\
\hline 26 & $25-26$ & 0 & - & - & - \\
\hline 27 & $26-27$ & 0 & - & - & - \\
\hline 28 & $27-28$ & 0 & - & - & - \\
\hline 29 & $28-29$ & 0 & - & - & - \\
\hline 30 & $29-30$ & 0 & - & - & - \\
\hline & TOTAL & 200 & & & \\
\hline
\end{tabular}

\begin{tabular}{|c|c|c|c|c|c|}
12 & $11-12$ & 0 & - & - & - \\
\hline 13 & $12-13$ & 1 & 0.008 & 0.968 & $960-967$ \\
\hline 14 & $13-14$ & 0 & - & - & - \\
\hline 15 & $14-15$ & 0 & - & - & - \\
\hline 16 & $15-16$ & 0 & - & - & - \\
\hline 17 & $16-17$ & 2 & 0.016 & 0.984 & $968-983$ \\
\hline 18 & $17-18$ & 0 & - & - & - \\
\hline 19 & $18-19$ & 0 & - & - & - \\
\hline 20 & $19-20$ & 1 & 0.008 & 0.992 & $984-991$ \\
\hline 21 & $20-21$ & 0 & - & - & - \\
\hline 22 & $21-22$ & 0 & - & - & - \\
\hline 23 & $22-23$ & 0 & - & - & - \\
\hline 24 & $23-24$ & 0 & - & - & - \\
\hline 25 & $24-25$ & 0 & - & - & - \\
\hline 26 & $25-26$ & 0 & - & - & - \\
\hline 27 & $26-27$ & 0 & - & - & - \\
\hline 28 & $27-28$ & 0 & - & - & - \\
\hline 29 & $28-29$ & 0 & - & - & - \\
\hline 30 & $29-30$ & 1 & 0.008 & 1 & $992-999$ \\
\hline & TOTAL & 125 & & & \\
\hline
\end{tabular}

\begin{tabular}{|c|c|c|c|c|c|}
\hline \multicolumn{7}{|c|}{ ARRIVAL DISTRIBUTION FOR F } \\
\hline $\begin{array}{c}\text { S. } \\
\text { No }\end{array}$ & $\begin{array}{c}\text { Inter } \\
\text { Arrival } \\
\text { Time(min) }\end{array}$ & $\begin{array}{c}\text { No. of } \\
\text { Vehicles }\end{array}$ & Prob. & $\begin{array}{c}\text { Cum. } \\
\text { Prob. }\end{array}$ & Tag Nos. \\
\hline 1 & $0-1$ & 23 & 0.211 & 0.211 & $000-210$ \\
\hline 2 & $1-2$ & 25 & 0.229 & 0.44 & $211-439$ \\
\hline 3 & $2-3$ & 12 & 0.11 & 0.55 & $440-549$ \\
\hline 4 & $3-4$ & 12 & 0.11 & 0.66 & $550-659$ \\
\hline 5 & $4-5$ & 11 & 0.101 & 0.761 & $660-760$ \\
\hline 6 & $5-6$ & 8 & 0.073 & 0.834 & $761-833$ \\
\hline 7 & $6-7$ & 6 & 0.056 & 0.89 & $834-889$ \\
\hline 8 & $7-8$ & 2 & 0.018 & 0.908 & $890-907$ \\
\hline 9 & $8-9$ & 1 & 0.009 & 0.917 & $908-916$ \\
\hline 10 & $9-10$ & 2 & 0.018 & 0.935 & $917-934$ \\
\hline 11 & $10-11$ & 0 & - & - & - \\
\hline 12 & $11-12$ & 2 & 0.018 & 0.953 & $935-952$ \\
\hline 13 & $12-13$ & 0 & - & - & - \\
\hline 14 & $13-14$ & 0 & - & - & - \\
\hline 15 & $14-15$ & 1 & 0.01 & 0.963 & $953-962$ \\
\hline 16 & $15-16$ & 0 & - & - & - \\
\hline 17 & $16-17$ & 0 & - & - & - \\
\hline 18 & $17-18$ & 0 & - & - & - \\
\hline 19 & $18-19$ & 1 & 0.009 & 0.972 & $963-971$ \\
\hline 20 & $19-20$ & 1 & 0.009 & 0.981 & $972-980$ \\
\hline 21 & $20-21$ & 0 & - & - & - \\
\hline 22 & $21-22$ & 0 & - & - & - \\
\hline 23 & $22-23$ & 0 & - & - & - \\
\hline 24 & $23-24$ & 0 & - & - & - \\
\hline 25 & $24-25$ & 0 & - & - & - \\
\hline 26 & $25-26$ & 0 & - & - & - \\
\hline 27 & $26-27$ & 1 & 0.01 & 0.991 & $981-990$ \\
\hline 28 & $27-28$ & 1 & 0.009 & 1 & $991-999$ \\
\hline 29 & $28-29$ & 0 & - & - & - \\
\hline 30 & $29-30$ & 0 & - & - & - \\
\hline
\end{tabular}

\begin{tabular}{|c|c|c|c|c|c|}
\hline \multicolumn{6}{|c|}{ ARRIVAL DISTRIBUTION FOR $\mathrm{F}_{2}$} \\
\hline $\begin{array}{c}\text { S. } \\
\text { No. }\end{array}$ & $\begin{array}{c}\text { Inter } \\
\text { Arrival } \\
\text { time(min) }\end{array}$ & $\begin{array}{c}\text { No. Of } \\
\text { Vehicles }\end{array}$ & Prob. & $\begin{array}{c}\text { Cum. } \\
\text { Prob. }\end{array}$ & Tag Nos. \\
\hline 1 & $0-1$ & 27 & 0.216 & 0.216 & $000-215$ \\
\hline 2 & $1-2$ & 27 & 0.216 & 0.432 & $216-431$ \\
\hline 3 & $2-3$ & 25 & 0.2 & 0.632 & $432-631$ \\
\hline 4 & $3-4$ & 8 & 0.064 & 0.696 & $632-695$ \\
\hline 5 & $4-5$ & 12 & 0.096 & 0.792 & $696-791$ \\
\hline 6 & $5-6$ & 6 & 0.048 & 0.84 & $792-839$ \\
\hline 7 & $6-7$ & 9 & 0.072 & 0.912 & $\begin{array}{c}840- \\
911\end{array}$ \\
\hline 8 & $7-8$ & 2 & 0.016 & 0.928 & $912-$ \\
\hline 9 & $8-9$ & 3 & 0.024 & 0.952 & $928-951$ \\
\hline 10 & $9-10$ & 1 & 0.008 & 0.96 & $952-$ \\
\hline 11 & $10-11$ & 0 & - & - & - \\
\hline
\end{tabular}


\begin{tabular}{|l|l|} 
TOTAL & 109 \\
\hline
\end{tabular}

\begin{tabular}{|c|c|c|c|c|c|}
\hline \multicolumn{6}{|c|}{ ARRIVAL DISTRIBUTION FOR F 4} \\
\hline $\begin{array}{c}\text { S. } \\
\text { No. }\end{array}$ & $\begin{array}{c}\text { Inter } \\
\text { Arrival } \\
\text { Time(min) }\end{array}$ & $\begin{array}{c}\text { No. of } \\
\text { Vehicles }\end{array}$ & Prob. & $\begin{array}{c}\text { Cum. } \\
\text { Prob. }\end{array}$ & Tag Nos. \\
\hline 1 & $0-1$ & 12 & 0.15 & 0.15 & $000-149$ \\
\hline 2 & $1-2$ & 11 & 0.138 & 0.288 & $150-287$ \\
\hline 3 & $2-3$ & 9 & 0.112 & 0.4 & $288-399$ \\
\hline 4 & $3-4$ & 12 & 0.15 & 0.55 & $400-549$ \\
\hline 5 & $4-5$ & 7 & 0.088 & 0.638 & $550-637$ \\
\hline 6 & $5-6$ & 7 & 0.088 & 0.726 & $638-725$ \\
\hline 7 & $6-7$ & 7 & 0.088 & 0.814 & $726-813$ \\
\hline 8 & $7-8$ & 3 & 0.037 & 0.851 & $814-850$ \\
\hline 9 & $8-9$ & 1 & 0.013 & 0.864 & $851-863$ \\
\hline 10 & $9-10$ & 0 & - & - & - \\
\hline 11 & $10-11$ & 0 & - & - & - \\
\hline 12 & $11-12$ & 2 & 0.025 & 0.889 & $864-888$ \\
\hline 13 & $12-13$ & 2 & 0.024 & 0.913 & $889-912$ \\
\hline
\end{tabular}

\begin{tabular}{|c|c|c|c|c|c|}
14 & $13-14$ & 0 & - & - & - \\
\hline 15 & $14-15$ & 0 & - & - & - \\
\hline 16 & $15-16$ & 0 & - & - & - \\
\hline 17 & $16-17$ & 0 & - & - & - \\
\hline 18 & $17-18$ & 3 & 0.037 & 0.95 & $913-949$ \\
\hline 19 & $18-19$ & 0 & - & - & - \\
\hline 20 & $19-20$ & 1 & 0.013 & 0.963 & $950-962$ \\
\hline 21 & $20-21$ & 0 & - & - & - \\
\hline 22 & $21-22$ & 1 & 0.012 & 0.975 & $963-974$ \\
\hline 23 & $22-23$ & 0 & - & - & - \\
\hline 24 & $23-24$ & 1 & 0.013 & 0.988 & $975-987$ \\
\hline 25 & $24-25$ & 0 & - & - & - \\
\hline 26 & $25-26$ & 0 & - & - & - \\
\hline 27 & $26-27$ & 1 & 0.012 & 1 & $988-999$ \\
\hline 28 & $27-28$ & 0 & - & - & - \\
\hline 29 & $28-29$ & 0 & - & - & - \\
\hline 30 & $29-30$ & 0 & - & - & - \\
\hline \multicolumn{7}{|l}{ TOTAL } & 80 & & & \\
\cline { 2 - 3 }
\end{tabular}

\section{Service Distribution Tables for $F_{1}, F_{2}, F_{3}, F_{4}$}

Service distribution for $F_{1}$

\begin{tabular}{|c|c|c|c|c|c|}
\hline S. No. & Service Time (min) & No. of Vehicles & Prob. & Cum. Prob. & Tag Nos. \\
\hline 1 & 1 & 92 & 0.46 & 0.46 \\
\hline 2 & 1.5 & 46 & 0.23 & 0.69 \\
\hline 3 & 2 & 26 & 0.13 & 0.82 & $460-689$ \\
\hline 4 & 2.5 & 20 & 0.1 & $69-819$ \\
\hline 5 & 3 & 16 & 0.08 & $820-919$ \\
\hline
\end{tabular}

Service distribution for $\mathrm{F}_{2}$

\begin{tabular}{|c|c|c|c|c|c|}
\hline S. No. & Service Time (min) & No. of Vehicles & Prob. & Cum. Prob. & Tag nos. \\
\hline 1 & 1 & 41 & 0.328 & 0.328 & $000-327$ \\
\hline 2 & 1.5 & 32 & 0.256 & 0.584 & $328-583$ \\
\hline 3 & 2 & 22 & 0.176 & 0.76 & $584-759$ \\
\hline 4 & 2.5 & 14 & 0.112 & 0.872 & $760-871$ \\
\hline \multirow[t]{2}{*}{5} & 3 & 16 & 0.128 & 1 & $872-999$ \\
\hline & Total & 125 & & & \\
\hline
\end{tabular}

Service distribution for $\mathrm{F}_{3}$

\begin{tabular}{|c|c|c|c|c|c|}
\hline S. No. & Service Time (min) & No. of Vehicles & Prob. & Cum. Prob. & Tag Nos. \\
\hline 1 & 1 & 34 & 0.312 & 0.312 & $000-311$ \\
\hline 2 & 1.5 & 26 & 0.239 & 0.551 & $312-550$ \\
\hline 3 & 2 & 20 & 0.183 & 0.734 & $551-733$ \\
\hline 4 & 2.5 & 11 & 0.101 & 0.835 & $734-834$ \\
\hline \multirow[t]{2}{*}{5} & 3 & 18 & 0.165 & 1 & $835-999$ \\
\hline & Total & 109 & & & \\
\hline
\end{tabular}

Service distribution for $\mathrm{F}_{4}$

\begin{tabular}{|c|c|c|c|c|c|}
\hline S. No. & Service Time (min) & No. of Vehicles & Prob. & Cum. Prob. & Tag Nos. \\
\hline 1 & 1 & 26 & 0.325 & 0.325 & $000-324$ \\
\hline 3 & 2 & 10 & 0.125 & 0.625 & $500-624$ \\
\hline 4 & 2.5 & 22 & 0.275 & 0.9 & $625-899$ \\
\hline
\end{tabular}




\section{Calculation-Simulation Tables for $F_{1}, F_{2}, F_{3}, F_{4}$}

\begin{tabular}{|c|c|c|c|c|c|c|c|c|c|c|}
\hline \multirow{2}{*}{$\begin{array}{l}\text { S. } \\
\text { No }\end{array}$} & \multirow{2}{*}{$\begin{array}{c}\text { Random } \\
\text { No. }\end{array}$} & \multirow{2}{*}{$\begin{array}{c}\text { Inter } \\
\text { Arrival } \\
\text { Time } \\
(\mathrm{min}) \\
\end{array}$} & \multirow{2}{*}{$\begin{array}{l}\text { Actual } \\
\text { Arrival Time } \\
\quad(\min )\end{array}$} & \multirow{2}{*}{$\begin{array}{c}\text { Random } \\
\text { No. }\end{array}$} & \multirow{2}{*}{$\begin{array}{l}\text { Service } \\
\text { Begins }\end{array}$} & \multirow{2}{*}{$\begin{array}{l}\text { Service } \\
\text { Time } \\
(\min )\end{array}$} & \multirow{2}{*}{$\begin{array}{l}\text { Service } \\
\text { Ends }\end{array}$} & \multicolumn{2}{|c|}{ Waiting time } & \multirow{2}{*}{$\begin{array}{l}\text { Queue } \\
\text { Length }\end{array}$} \\
\hline & & & & & & & & Customer & Server & \\
\hline 1 & 218 & 1 & $10: 31: 00$ & 741 & $10: 31: 00$ & 2 & 10:33:00 & - & - & - \\
\hline 2 & 112 & 1 & $10: 32: 00$ & 766 & 10:33:00 & 2 & $10: 35: 00$ & 00:01:00 & - & 1 \\
\hline 3 & 711 & 3 & $10: 35: 00$ & 27 & $10: 35: 00$ & 1 & $10: 36: 00$ & - & - & - \\
\hline 4 & 655 & 3 & 10:38:00 & 70 & $10: 38: 00$ & 1 & 10:39:00 & - & 00:02:00 & - \\
\hline 5 & 419 & 1 & 10:39:00 & 648 & 10:39:00 & 1.5 & $10: 40: 30$ & - & - & - \\
\hline 6 & 354 & 1 & 10:40:00 & 956 & $10: 40: 30$ & 3 & $10: 43: 30$ & $00: 00: 30$ & - & 1 \\
\hline 7 & 174 & 1 & $10: 41: 00$ & 238 & $10: 43: 30$ & 1 & $10: 44: 30$ & $00: 02: 30$ & - & 1 \\
\hline 8 & 910 & 5 & $10: 46: 00$ & 912 & $10: 46: 00$ & 2.5 & $10: 48: 30$ & - & 00:01:30 & - \\
\hline 9 & 76 & 1 & $10: 47: 00$ & 480 & $10: 48: 30$ & 1.5 & 10:50:00 & $00: 01: 30$ & - & 1 \\
\hline 10 & 349 & 1 & $10: 48: 00$ & 558 & $10: 50: 00$ & 1.5 & $10: 51: 30$ & 00:02:00 & - & 1 \\
\hline 11 & 129 & 1 & $10: 49: 00$ & 917 & $10: 51: 30$ & 2.5 & $10: 54: 00$ & $00: 02: 30$ & - & 1 \\
\hline 12 & 439 & 2 & 10:51:00 & 403 & $10: 54: 00$ & 1 & 10:55:00 & 00:03:00 & - & 1 \\
\hline 13 & 380 & 1 & $10: 52: 00$ & 932 & $10: 55: 00$ & 3 & 10:58:00 & $00: 03: 00$ & - & 1 \\
\hline 14 & 498 & 2 & $10: 54: 00$ & 13 & $10: 58: 00$ & 1 & $10: 59: 00$ & 00:04:00 & - & 1 \\
\hline 15 & 134 & 1 & $10: 55: 00$ & 838 & 10:59:00 & 2.5 & 11:01:30 & 00:04:00 & - & 1 \\
\hline 16 & 59 & 1 & $10: 56: 00$ & 635 & $11: 01: 30$ & 1.5 & 11:03:00 & $00: 05: 30$ & - & 1 \\
\hline 17 & 966 & 8 & 11:04:00 & 476 & 11:04:00 & 1.5 & 11:05:30 & - & 00:01:00 & - \\
\hline 18 & 761 & 3 & 11:07:00 & 525 & 11:07:00 & 1.5 & 11:08:30 & - & $00: 01: 30$ & - \\
\hline 19 & 850 & 4 & 11:11:00 & 472 & $11: 11: 00$ & 1.5 & $11: 12: 30$ & - & $00: 02: 30$ & - \\
\hline 20 & 697 & 3 & $11: 14: 00$ & 364 & $11: 14: 00$ & 1 & 11:15:00 & - & $00: 01: 30$ & - \\
\hline 21 & 579 & 2 & 11:16:00 & 576 & $11: 16: 00$ & 1.5 & $11: 17: 30$ & - & 00:01:00 & - \\
\hline 22 & 636 & 2 & $11: 18: 00$ & 44 & $11: 18: 00$ & 1 & $11: 19: 00$ & - & $00: 00: 30$ & - \\
\hline 23 & 416 & 1 & 11:19:00 & 793 & 11:19:00 & 2 & 11:21:00 & - & - & - \\
\hline 24 & 35 & 1 & 11:20:00 & 553 & $11: 21: 00$ & 1.5 & $11: 22: 30$ & 00:01:00 & - & 1 \\
\hline 25 & 913 & 6 & $11: 26: 00$ & 105 & $11: 26: 00$ & 1 & $11: 27: 00$ & - & $00: 03: 30$ & - \\
\hline 26 & 582 & 2 & $11: 28: 00$ & 133 & $11: 28: 00$ & 1 & $11: 29: 00$ & - & 00:01:00 & - \\
\hline 27 & 628 & 2 & 11:30:00 & 572 & 11:30:00 & 1.5 & $11: 31: 30$ & - & 00:01:00 & - \\
\hline 28 & 752 & 3 & 11:33:00 & 90 & $11: 33: 00$ & 1 & $11: 34: 00$ & - & $00: 01: 30$ & - \\
\hline 29 & 897 & 5 & 11:38:00 & 163 & $11: 38: 00$ & 1 & 11:39:00 & - & 00:04:00 & - \\
\hline 30 & 232 & 1 & 11:39:00 & 734 & 11:39:00 & 2 & 11:41:00 & - & - & - \\
\hline 31 & 218 & 1 & 11:40:00 & 280 & $11: 41: 00$ & 1 & 11:42:00 & 00:01:00 & - & 1 \\
\hline 32 & 365 & 1 & 11:41:00 & 74 & $11: 42: 00$ & 1 & 11:43:00 & 00:01:00 & - & 1 \\
\hline 33 & 592 & 2 & $11: 43: 00$ & 304 & $11: 43: 00$ & 1 & $11: 44: 00$ & - & - & - \\
\hline 34 & 394 & 1 & $11: 44: 00$ & 620 & $11: 44: 00$ & 1.5 & $11: 45: 30$ & - & - & - \\
\hline 35 & 195 & 1 & $11: 45: 00$ & 292 & $11: 45: 30$ & 1 & $11: 46: 30$ & $00: 00: 30$ & - & 1 \\
\hline 36 & 211 & 1 & 11:46:00 & 669 & $11: 46: 30$ & 1.5 & 11:48:00 & 00:00:30 & - & 1 \\
\hline 37 & 747 & 3 & 11:49:00 & 826 & $11: 49: 00$ & 2.5 & $11: 51: 30$ & - & 00:01:00 & - \\
\hline 38 & 867 & 5 & $11: 54: 00$ & 958 & $11: 54: 00$ & 3 & $11: 57: 00$ & - & $00: 02: 30$ & - \\
\hline 39 & 906 & 5 & 11:59:00 & 188 & 11:59:00 & 1 & 12:00:00 & - & $00: 02: 00$ & - \\
\hline 40 & 643 & 2 & 12:01:00 & 967 & 12:01:00 & 3 & 12:04:00 & - & 00:01:00 & - \\
\hline 41 & 183 & 1 & $12: 02: 00$ & 203 & $12: 04: 00$ & 1 & $12: 05: 00$ & $00: 02: 00$ & - & 1 \\
\hline 42 & 188 & 1 & 12:03:00 & 841 & $12: 05: 00$ & 2.5 & $12: 07: 30$ & 00:02:00 & - & 1 \\
\hline 43 & 679 & 3 & 12:06:00 & 567 & 12:07:30 & 1.5 & 12:09:00 & 00:01:30 & - & 1 \\
\hline 44 & 206 & 1 & 12:07:00 & 119 & 12:09:00 & 1 & 12:10:00 & 00:02:00 & - & 1 \\
\hline 45 & 729 & 3 & 12:10:00 & 526 & $12: 10: 00$ & 1.5 & $12: 11: 30$ & - & - & - \\
\hline 46 & 344 & 1 & 12:11:00 & 38 & $12: 11: 30$ & 1 & $12: 12: 30$ & 00:00:30 & - & 1 \\
\hline 47 & 543 & 2 & 12:13:00 & 516 & $12: 13: 00$ & 1.5 & $12: 14: 30$ & - & $00: 00: 30$ & - \\
\hline 48 & 309 & 1 & $12: 14: 00$ & 403 & $12: 14: 30$ & 1 & $12: 15: 30$ & $00: 00: 30$ & - & 1 \\
\hline 49 & 227 & 1 & $12: 15: 00$ & 345 & $12: 15: 30$ & 1 & $12: 16: 30$ & 00:00:30 & - & 1 \\
\hline 50 & 483 & 2 & $12: 17: 00$ & 385 & $12: 17: 00$ & 1 & 12:18:00 & - & $00: 00: 30$ & - \\
\hline & & 107 & & & & 77 & & $00: 42: 30$ & $00: 30: 00$ & 23 \\
\hline
\end{tabular}

1. Average queue length $=23 / 50=0.46$.

2. Average waiting time of a customer $=42.5 / 50=0.85$ minutes.

3. Average service time $=77 / 50=1.54$ minutes.

4. Time A customer spends in the system $=0.85+1.54=2.39$ minutes.

5. Average waiting time of a server $=30 / 50=0.6$ minutes.

6. Average arrival time $=107 / 50=2.14$ minutes . 
A Simulation Study on toll gate system in M/M/1 Queueing Models

\begin{tabular}{|c|c|c|c|c|c|c|c|c|c|c|}
\hline \multicolumn{11}{|c|}{$\begin{array}{ll}\text { SIMULATION TABLE FOR F2 } \\
\end{array}$} \\
\hline \multirow[b]{2}{*}{$\begin{array}{l}\text { S. } \\
\text { No }\end{array}$} & \multirow[b]{2}{*}{$\begin{array}{c}\text { Random } \\
\text { No. }\end{array}$} & \multirow{2}{*}{$\begin{array}{l}\text { Inter } \\
\text { Arrival } \\
\text { Time } \\
(\mathrm{min})\end{array}$} & \multirow{2}{*}{$\begin{array}{c}\text { Actual } \\
\text { Arrival Time } \\
\quad(\mathrm{min})\end{array}$} & \multirow[b]{2}{*}{$\begin{array}{c}\text { Random } \\
\text { No. }\end{array}$} & \multirow[b]{2}{*}{$\begin{array}{l}\text { Service } \\
\text { Begins }\end{array}$} & \multirow{2}{*}{$\begin{array}{l}\text { Service } \\
\text { Time } \\
(\mathrm{min})\end{array}$} & \multirow[b]{2}{*}{$\begin{array}{l}\text { Service } \\
\text { Ends }\end{array}$} & \multicolumn{2}{|c|}{ Waiting time } & \multirow[b]{2}{*}{$\begin{array}{l}\text { Queue } \\
\text { Length }\end{array}$} \\
\hline & & & & & & & & Customer & Server & \\
\hline 1 & 722 & 5 & 10:48:00 & 232 & 10:48:00 & 1 & 10:49:00 & - & - & - \\
\hline 2 & 116 & 1 & 10:49:00 & 457 & 10:49:00 & 1.5 & $10: 50: 30$ & - & - & - \\
\hline 3 & 508 & 3 & $10: 52: 00$ & 546 & $10: 52: 00$ & 1.5 & $10: 53: 30$ & - & 00:01:30 & - \\
\hline 4 & 554 & 1 & 10:53:00 & 248 & $10: 53: 30$ & 1 & $10: 54: 30$ & $00: 00: 30$ & - & 1 \\
\hline 5 & 84 & 1 & $10: 54: 00$ & 504 & $10: 54: 30$ & 1.5 & $10: 56: 00$ & 00:00:30 & - & 1 \\
\hline 6 & 118 & 1 & $10: 55: 00$ & 292 & $10: 56: 00$ & 1 & $10: 57: 00$ & 00:01:00 & - & 1 \\
\hline 7 & 767 & 5 & 11:00:00 & 618 & 11:00:00 & 2 & 11:02:00 & - & 00:03:00 & \\
\hline 8 & 189 & 1 & 11:01:00 & 220 & 11:02:00 & 1 & 11:03:00 & 00:01:00 & - & 1 \\
\hline 9 & 338 & 2 & 11:03:00 & 471 & 11:03:00 & 1.5 & 11:04:30 & - & - & - \\
\hline 10 & 814 & 6 & 11:09:00 & 627 & 11:09:00 & 2 & 11:11:00 & - & 00:04:30 & - \\
\hline 11 & 351 & 2 & 11:11:00 & 823 & 11:11:00 & 2.5 & $11: 13: 30$ & - & - & - \\
\hline 12 & 964 & 13 & $11: 24: 00$ & 527 & $11: 24: 00$ & 1.5 & $11: 25: 30$ & - & $00: 10: 30$ & - \\
\hline 13 & 843 & 7 & 11:31:00 & 182 & $11: 31: 00$ & 1 & 11:32:00 & - & $00: 05: 30$ & - \\
\hline 14 & 876 & 7 & 11:38:00 & 638 & 11:38:00 & 2 & 11:40:00 & - & 00:06:00 & - \\
\hline 15 & 874 & 7 & $11: 45: 00$ & 147 & $11: 45: 00$ & 1 & 11:46:00 & - & 00:05:00 & - \\
\hline 16 & 952 & 10 & 11:55:00 & 320 & 11:55:00 & 1 & 11:56:00 & - & 00:09:00 & - \\
\hline 17 & 476 & 3 & 11:58:00 & 822 & 11:58:00 & 2.5 & 12:00:30 & - & 00:02:00 & - \\
\hline 18 & 841 & 7 & 12:05:00 & 633 & 12:05:00 & 2 & 12:07:00 & - & 00:04:30 & - \\
\hline 19 & 943 & 9 & 12:14:00 & 548 & 12:14:00 & 1.5 & $12: 15: 30$ & - & 00:07:00 & - \\
\hline 20 & 342 & 2 & 12:16:00 & 344 & 12:16:00 & 1.5 & 12:17:30 & - & 00:00:30 & - \\
\hline 21 & 682 & 4 & $12: 20: 00$ & 461 & $12: 20: 00$ & 1.5 & $12: 21: 30$ & - & $00: 02: 30$ & - \\
\hline 22 & 852 & 7 & $12: 27: 00$ & 319 & $12: 27: 00$ & 1 & $12: 28: 00$ & - & $00: 05: 30$ & - \\
\hline 23 & 112 & 1 & $12: 28: 00$ & 483 & $12: 28: 00$ & 1.5 & $12: 29: 30$ & - & - & - \\
\hline 24 & 580 & 3 & 12:31:00 & 517 & 12:31:00 & 1.5 & 12:32:30 & - & 00:01:30 & - \\
\hline 25 & 928 & 9 & 12:40:00 & 63 & $12: 40: 00$ & 1 & 12:41:00 & - & $00: 07: 30$ & - \\
\hline 26 & 695 & 4 & 12:44:00 & 229 & 12:44:00 & 1 & $12: 45: 00$ & - & 00:03:00 & - \\
\hline 27 & 593 & 3 & $12: 47: 00$ & 797 & 12:47:00 & 2.5 & 12:49:30 & - & 00:02:00 & - \\
\hline 28 & 804 & 6 & $12: 53: 00$ & 562 & $12: 53: 00$ & 1.5 & $12: 54: 30$ & - & $00: 03: 30$ & - \\
\hline 29 & 221 & 2 & $12: 55: 00$ & 66 & $12: 55: 00$ & 1 & $12: 56: 00$ & - & 00:00:30 & - \\
\hline 30 & 837 & 6 & 1:01:00 & 914 & 01:01:00 & 3 & 01:04:00 & - & 00:05:00 & - \\
\hline 31 & 705 & 5 & 1:06:00 & 511 & 01:06:00 & 1.5 & 01:07:30 & - & 00:02:00 & - \\
\hline 32 & 68 & 1 & $1: 07: 00$ & 134 & 01:07:30 & 1 & $01: 08: 30$ & 00:00:30 & - & 1 \\
\hline 33 & 129 & 1 & 1:08:00 & 657 & 01:08:30 & 2 & 01:10:30 & 00:00:30 & - & 1 \\
\hline 34 & 597 & 3 & 1:11:00 & 602 & $01: 11: 00$ & 2 & 01:13:00 & - & 00:00:30 & - \\
\hline 35 & 467 & 3 & $1: 14: 00$ & 511 & $01: 14: 00$ & 1.5 & $01: 15: 30$ & - & 00:01:00 & - \\
\hline 36 & 543 & 3 & $1: 17: 00$ & 504 & 01:17:00 & 1.5 & 01:18:30 & - & 00:01:30 & - \\
\hline 37 & 41 & 1 & $1: 18: 00$ & 132 & $01: 18: 30$ & 1 & $01: 19: 30$ & 00:00:30 & - & 1 \\
\hline 38 & 30 & 3 & $1: 21: 00$ & 946 & $01: 21: 00$ & 3 & $01: 24: 00$ & - & $00: 01: 30$ & - \\
\hline 39 & 999 & 30 & 1:51:00 & 579 & $01: 51: 00$ & 1.5 & $01: 52: 30$ & - & $00: 27: 00$ & - \\
\hline 40 & 840 & 7 & 1:58:00 & 262 & 01:58:00 & 1 & 01:59:00 & - & 00:05:30 & - \\
\hline 41 & 812 & 6 & $2: 04: 00$ & 787 & 02:04:00 & 2.5 & $02: 06: 30$ & - & 00:05:00 & - \\
\hline 42 & 153 & 1 & 2:05:00 & 331 & $02: 06: 30$ & 1.5 & 02:08:00 & 00:01:30 & - & 1 \\
\hline 43 & 364 & 2 & $2: 07: 00$ & 600 & 02:08:00 & 2 & $02: 10: 00$ & 00:01:00 & - & 1 \\
\hline 44 & 126 & 1 & $2: 08: 00$ & 318 & $02: 10: 00$ & 1 & $02: 11: 00$ & $00: 02: 00$ & - & 1 \\
\hline 45 & 540 & 3 & $2: 11: 00$ & 151 & $02: 11: 00$ & 1 & $02: 12: 00$ & - & - & - \\
\hline 46 & 978 & 17 & $2: 28: 00$ & 647 & $02: 28: 00$ & 2 & 02:30:00 & - & 00:16:00 & - \\
\hline 47 & 1 & 1 & $2: 29: 00$ & 890 & 02:30:00 & 3 & 02:33:00 & 00:01:00 & - & 1 \\
\hline 48 & 494 & 3 & $2: 32: 00$ & 746 & $02: 33: 00$ & 2 & $02: 35: 00$ & $00: 01: 00$ & - & 1 \\
\hline 49 & 442 & 3 & $2: 35: 00$ & 995 & $02: 35: 00$ & 3 & $02: 38: 00$ & - & - & - \\
\hline \multirow[t]{2}{*}{50} & 135 & 1 & $2: 36: 00$ & 636 & $02: 38: 00$ & 2 & $02: 40: 00$ & 00:02:00 & - & 1 \\
\hline & & 235 & & & & 82 & & $00: 13: 00$ & $02: 29: 30$ & 13 \\
\hline
\end{tabular}

1. Average queue length $=13 / 50=0.26$

2. Average waiting time of a customer $=13 / 50=0.26$ minutes.

3. Average service time $=82 / 50=1.64$ minutes.

4. Time a customer spends in the system $=0.26+1.64=1.9$ minutes

5. Average waiting time of a server $=149.5 / 50=2.99$ minutes.

6. Average arrival time $=235 / 50=4.7$ minutes. 
SIMULATION TABLE FOR F3

\begin{tabular}{|c|c|c|c|c|c|c|c|c|c|c|}
\hline \multirow{2}{*}{$\begin{array}{l}\text { S. } \\
\text { No }\end{array}$} & \multirow{2}{*}{$\begin{array}{c}\text { Random } \\
\text { No. }\end{array}$} & \multirow{2}{*}{$\begin{array}{c}\text { Inter } \\
\text { Arrival } \\
\text { Time } \\
(\mathrm{min})\end{array}$} & \multirow{2}{*}{$\begin{array}{l}\text { Actual } \\
\text { Arrival } \\
\text { Time } \\
(\mathrm{min})\end{array}$} & \multirow{2}{*}{$\begin{array}{c}\text { Random } \\
\text { No. }\end{array}$} & \multirow{2}{*}{$\begin{array}{l}\text { Service } \\
\text { Begins }\end{array}$} & \multirow{2}{*}{$\begin{array}{l}\text { Service } \\
\text { Time } \\
(\min )\end{array}$} & \multirow{2}{*}{$\begin{array}{c}\text { Service } \\
\text { Ends }\end{array}$} & \multicolumn{2}{|c|}{ Waiting time } & \multirow{2}{*}{$\begin{array}{l}\text { Queue } \\
\text { Length }\end{array}$} \\
\hline & & & & & & & & Customer & Server & \\
\hline 1 & 589 & 4 & 10:47:00 & 759 & $10: 47: 00$ & 2.5 & $10: 49: 30$ & - & - & - \\
\hline 2 & 830 & 6 & 10:53:00 & 156 & 10:53:00 & 1 & $10: 54: 00$ & - & 00:03:30 & - \\
\hline 3 & 444 & 3 & 10:56:00 & 426 & 10:56:00 & 1.5 & $10: 57: 30$ & - & 00:02:00 & - \\
\hline 4 & 642 & 4 & 11:00:00 & 112 & 11:00:00 & 1 & 11:01:00 & - & $00: 02: 30$ & - \\
\hline 5 & 590 & 4 & 11:04:00 & 853 & 11:04:00 & 3 & 11:07:00 & - & 00:03:00 & - \\
\hline 6 & 31 & 1 & 11:05:00 & 418 & 11:07:00 & 1.5 & 11:08:30 & 00:02:00 & - & 1 \\
\hline 7 & 590 & 4 & 11:09:00 & 422 & 11:09:00 & 1.5 & $11: 10: 30$ & - & $00: 00: 30$ & - \\
\hline 8 & 304 & 2 & 11:11:00 & 185 & 11:11:00 & 1 & 11:12:00 & - & $00: 00: 30$ & - \\
\hline 9 & 169 & 1 & 11:12:00 & 857 & 11:12:00 & 3 & 11:15:00 & - & - & - \\
\hline 10 & 573 & 4 & 11:16:00 & 508 & 11:16:00 & 1.5 & $11: 17: 30$ & - & 00:01:00 & - \\
\hline 11 & 876 & 7 & $11: 23: 00$ & 67 & 11:23:00 & 1 & $11: 24: 00$ & - & 00:05:30 & - \\
\hline 12 & 216 & 2 & $11: 25: 00$ & 581 & $11: 25: 00$ & 3 & 11:28:00 & - & 00:01:00 & - \\
\hline 13 & 369 & 2 & $11: 27: 00$ & 90 & $11: 28: 00$ & 1 & 11:29:00 & 00:01:00 & - & 1 \\
\hline 14 & 607 & 4 & $11: 31: 00$ & 569 & 11:31:00 & 2 & $11: 33: 00$ & - & 00:02:00 & - \\
\hline 15 & 822 & 6 & $11: 37: 00$ & 263 & $11: 37: 00$ & 1 & 11:38:00 & - & 00:04:00 & - \\
\hline 16 & 192 & 1 & 11:38:00 & 368 & $11: 38: 00$ & 1.5 & $11: 39: 30$ & - & - & - \\
\hline 17 & 810 & 6 & $11: 44: 00$ & 612 & $11: 44: 00$ & 2 & $11: 46: 00$ & - & $00: 04: 30$ & - \\
\hline 18 & 207 & 1 & $11: 45: 00$ & 275 & 11:46:00 & 1 & $11: 47: 00$ & 00:01:00 & - & 1 \\
\hline 19 & 747 & 5 & 11:50:00 & 1 & 11:50:00 & 1 & 11:51:00 & - & 00:03:00 & - \\
\hline 20 & 952 & 12 & $12: 02: 00$ & 888 & $12: 02: 00$ & 3 & $12: 05: 00$ & - & $00: 11: 00$ & - \\
\hline 21 & 517 & 4 & 12:06:00 & 426 & 12:06:00 & 1.5 & $12: 07: 30$ & 00:01:00 & & 1 \\
\hline 22 & 942 & 12 & $12: 18: 00$ & 927 & 12:18:00 & 3 & $12: 21: 00$ & - & $00: 10: 30$ & - \\
\hline 23 & 316 & 2 & $12: 20: 00$ & 83 & $12: 21: 00$ & 1 & $12: 22: 00$ & 00:01:00 & & 1 \\
\hline 24 & 423 & 2 & $12: 22: 00$ & 8 & $12: 22: 00$ & 1 & $12: 23: 00$ & - & - & - \\
\hline 25 & 396 & 2 & $12: 24: 00$ & 526 & $12: 24: 00$ & 1.5 & $12: 25: 30$ & - & 00:01:00 & - \\
\hline 26 & 205 & 1 & $12: 25: 00$ & 282 & $12: 25: 30$ & 1 & $12: 26: 30$ & 00:00:30 & & 1 \\
\hline 27 & 742 & 5 & $12: 30: 00$ & 555 & $12: 30: 00$ & 2 & $12: 32: 00$ & - & $00: 03: 30$ & - \\
\hline 28 & 795 & 6 & $12: 36: 00$ & 473 & $12: 36: 00$ & 1.5 & $12: 37: 30$ & - & 00:04:00 & - \\
\hline 29 & 476 & 4 & $12: 40: 00$ & 596 & 12:40:00 & 2 & $12: 42: 00$ & - & $00: 02: 30$ & - \\
\hline 30 & 14 & 1 & $12: 41: 00$ & 323 & $12: 42: 00$ & 1.5 & $12: 43: 30$ & 00:01:00 & - & 1 \\
\hline 31 & 45 & 1 & $12: 42: 00$ & 910 & $12: 43: 30$ & 3 & $12: 46: 30$ & 00:01:30 & - & 1 \\
\hline 32 & 655 & 4 & $12: 46: 00$ & 510 & $12: 46: 30$ & 1.5 & $12: 48: 00$ & 00:00:30 & - & 1 \\
\hline 33 & 511 & 4 & $12: 50: 00$ & 514 & 12:50:00 & 1.5 & $12: 51: 30$ & - & 00:02:00 & - \\
\hline 34 & 877 & 7 & $12: 57: 00$ & 537 & $12: 57: 00$ & 1.5 & $12: 58: 30$ & - & $00: 05: 30$ & - \\
\hline 35 & 2 & 1 & $12: 58: 00$ & 612 & $12: 58: 30$ & 2 & $1: 00: 30$ & $00: 00: 30$ & - & 1 \\
\hline 36 & 999 & 28 & $1: 26: 00$ & 555 & $1: 26: 00$ & 2 & $1: 28: 00$ & - & $00: 25: 30$ & - \\
\hline 37 & 437 & 2 & $1: 28: 00$ & 160 & 1:28:00 & 1 & 1:29:00 & - & - & - \\
\hline 38 & 549 & 4 & $1: 32: 00$ & 679 & $1: 32: 00$ & 2 & $1: 34: 00$ & - & 00:03:00 & - \\
\hline 39 & 468 & 4 & $1: 36: 00$ & 579 & 1:36:00 & 2 & $1: 38: 00$ & - & 00:02:00 & - \\
\hline 40 & 55 & 1 & $1: 37: 00$ & 798 & 1:38:00 & 2.5 & $1: 40: 30$ & 00:01:00 & - & 1 \\
\hline 41 & 698 & 5 & $1: 42: 00$ & 375 & 1:42:00 & 1.5 & $1: 43: 30$ & - & $00: 01: 30$ & - \\
\hline 42 & 504 & 4 & $1: 46: 00$ & 628 & 1:46:00 & 2 & $1: 48: 00$ & - & 00:02:30 & - \\
\hline 43 & 779 & 6 & $1: 52: 00$ & 309 & 1:52:00 & 1 & 1:53:00 & - & 00:04:00 & - \\
\hline 44 & 647 & 4 & $1: 56: 00$ & 410 & 1:56:00 & 1.5 & $1: 57: 30$ & - & 00:03:00 & - \\
\hline 45 & 799 & 6 & $2: 02: 00$ & 461 & $2: 02: 00$ & 1.5 & $2: 03: 30$ & - & $00: 04: 30$ & - \\
\hline 46 & 209 & 1 & 2:03:00 & 945 & $2: 03: 30$ & 3 & $2: 06: 30$ & $00: 00: 30$ & - & 1 \\
\hline 47 & 939 & 10 & $2: 13: 00$ & 698 & $2: 13: 00$ & 2 & $2: 15: 00$ & - & 00:06:30 & - \\
\hline 48 & 477 & 4 & $2: 17: 00$ & 906 & $2: 17: 00$ & 3 & $2: 20: 00$ & - & 00:02:00 & - \\
\hline 49 & 109 & 1 & $2: 18: 00$ & 347 & $2: 20: 00$ & 1.5 & $2: 21: 30$ & 00:02:00 & 00:01:00 & 1 \\
\hline \multirow[t]{2}{*}{50} & 510 & 4 & $2: 22: 00$ & 372 & $2: 22: 00$ & 1.5 & $2: 23: 30$ & - & 00:00:30 & - \\
\hline & & 219 & & & & 87.5 & & 00:13:30 & 02:09:00 & 13 \\
\hline
\end{tabular}

1. Average Queue Length $=13 / 50=0.26$

2. Average waiting time of a customer $=13.5 / 50=0.27$ minutes

3. Average service time $=87.5 / 50=1.75$ minutes .

4. Time a customer spends in the system $=0.27+1.75=2.02$ minutes.

5. Average waiting time of a server $=129 / 50=4$ minutes.

6. Average arrival time $=219 / 50=4.4$ minutes. 
A Simulation Study on toll gate system in M/M/1 Queueing Models

\begin{tabular}{|c|c|c|c|c|c|c|c|c|c|c|}
\hline \multirow{3}{*}{$\begin{array}{l}\text { S. } \\
\text { No }\end{array}$} & \multirow{3}{*}{$\begin{array}{c}\text { Random } \\
\text { No. }\end{array}$} & \multirow{3}{*}{$\begin{array}{c}\text { Inter } \\
\text { Arrival } \\
\text { Time } \\
\text { (min) }\end{array}$} & \multicolumn{7}{|c|}{ SIMULATION TABLE FOR F4 } & \multirow{3}{*}{$\begin{array}{l}\text { Queue } \\
\text { Length }\end{array}$} \\
\hline & & & \multirow{2}{*}{$\begin{array}{l}\text { Actual } \\
\text { Arrival } \\
\text { Time } \\
\text { (min) }\end{array}$} & \multirow{2}{*}{$\begin{array}{c}\text { Random } \\
\text { No. }\end{array}$} & \multirow{2}{*}{$\begin{array}{l}\text { Service } \\
\text { Begins }\end{array}$} & \multirow{2}{*}{$\begin{array}{l}\text { Service } \\
\text { Time } \\
(\min )\end{array}$} & \multirow{2}{*}{$\begin{array}{l}\text { Service } \\
\text { Ends }\end{array}$} & \multicolumn{2}{|c|}{ Waiting Time } & \\
\hline & & & & & & & & Customer & Server & \\
\hline 1 & 624 & 5 & $10: 58: 00$ & 211 & $10: 58: 00$ & 1 & $10: 59: 00$ & - & - & - \\
\hline 2 & 213 & 2 & $10: 58: 00$ & 429 & $10: 58: 00$ & 1.5 & 11:01:30 & - & 00:01:00 & - \\
\hline 3 & 611 & 5 & 11:05:00 & 364 & $11: 05: 00$ & 1.5 & $11: 06: 30$ & - & $00: 03: 30$ & - \\
\hline 5 & 85 & 1 & 11:08:00 & 870 & $11: 10: 00$ & 2.5 & $11: 12: 30$ & 00:02:00 & - & 1 \\
\hline 6 & 959 & 20 & $11: 28: 00$ & 354 & $11: 28: 00$ & 1.5 & $11: 29: 30$ & - & $00: 15: 30$ & - \\
\hline 7 & 657 & 6 & $11: 34: 00$ & 102 & $11: 34: 00$ & 1 & $11: 35: 00$ & - & 00:04:30 & - \\
\hline 8 & 98 & 1 & $11: 35: 00$ & 376 & $11: 35: 00$ & 1.5 & $11: 36: 30$ & - & - & - \\
\hline 9 & 875 & 12 & $11: 47: 00$ & 717 & $11: 47: 00$ & 2.5 & $11: 49: 30$ & - & $00: 10: 30$ & - \\
\hline 10 & 309 & 3 & $11: 50: 00$ & 792 & $11: 50: 00$ & 2.5 & $11: 52: 30$ & - & $00: 00: 30$ & - \\
\hline 15 & 943 & 18 & $12: 40: 00$ & 103 & $12: 40: 00$ & 1 & $12: 41: 00$ & - & $00: 16: 00$ & - \\
\hline 16 & 856 & 9 & $12: 49: 00$ & 367 & $12: 49: 00$ & 1.5 & $12: 50: 30$ & - & 00:08:00 & - \\
\hline 17 & 562 & 5 & $12: 54: 00$ & 942 & $12: 54: 00$ & 3 & $12: 57: 00$ & - & $00: 03: 30$ & - \\
\hline 18 & 905 & 13 & 01:07:00 & 863 & 01:07:00 & 2.5 & $01: 09: 30$ & - & $00: 10: 00$ & - \\
\hline 19 & 481 & 4 & 01:11:00 & 167 & $01: 11: 00$ & 1 & 01:12:00 & - & $00: 01: 30$ & - \\
\hline 20 & 12 & 1 & $01: 12: 00$ & 908 & $01: 12: 00$ & 3 & $01: 15: 00$ & - & - & - \\
\hline 21 & 626 & 5 & 01:17:00 & 879 & 01:17:00 & 2.5 & $01: 19: 30$ & - & 00:02:00 & - \\
\hline 22 & 893 & 13 & $01: 30: 00$ & 567 & 01:30:00 & 2 & 01:32:00 & - & $00: 10: 30$ & - \\
\hline 23 & 285 & 2 & 01:32:00 & 227 & $01: 32: 00$ & 1 & 01:33:00 & - & - & - \\
\hline 24 & 256 & 2 & 01:34:00 & 187 & $01: 34: 00$ & 1 & 01:35:00 & - & 00:01:00 & - \\
\hline 33 & 660 & 6 & 02:54:00 & 198 & $02: 54: 00$ & 1 & $02: 55: 00$ & - & 00:04:30 & - \\
\hline 34 & 407 & 4 & 02:58:00 & 296 & 02:58:00 & 1 & 02:59:00 & - & 00:03:00 & - \\
\hline 35 & 84 & 1 & $02: 59: 00$ & 528 & $02: 59: 00$ & 2 & 03:01:00 & - & - & - \\
\hline 36 & 782 & 7 & 03:06:00 & 120 & 03:06:00 & 1 & 03:07:00 & - & 00:05:00 & - \\
\hline 37 & 105 & 1 & 03:07:00 & 388 & 03:07:00 & 1.5 & 03:08:30 & - & - & - \\
\hline 38 & 811 & 7 & $03: 14: 00$ & 765 & $03: 14: 00$ & 2.5 & $03: 16: 30$ & - & $00: 05: 30$ & - \\
\hline 39 & 655 & 6 & 03:20:00 & 238 & $03: 20: 00$ & 1 & $03: 21: 00$ & - & $00: 03: 30$ & - \\
\hline 40 & 530 & 4 & $03: 24: 00$ & 314 & $03: 24: 00$ & 1 & $03: 25: 00$ & - & 00:03:00 & - \\
\hline 41 & 515 & 4 & 03:28:00 & 3 & $03: 28: 00$ & 1 & $03: 29: 00$ & - & $00: 03: 00$ & - \\
\hline 42 & 780 & 7 & 03:35:00 & 613 & 03:35:00 & 2 & 03:37:00 & - & 00:06:00 & - \\
\hline 43 & 438 & 4 & $03: 39: 00$ & 936 & $03: 39: 00$ & 3 & $03: 42: 00$ & - & $00: 02: 00$ & - \\
\hline 44 & 959 & 20 & 03:59:00 & 609 & $03: 59: 00$ & 2 & 04:00:30 & - & $00: 17: 00$ & - \\
\hline 45 & 485 & 4 & 04:03:00 & 12 & 04:03:00 & 1 & 04:04:00 & - & $00: 20: 00$ & - \\
\hline 46 & 550 & 5 & 04:08:00 & 196 & 04:08:00 & 1 & 04:09:00 & - & 00:04:00 & - \\
\hline 47 & 401 & 4 & 04:12:00 & 405 & $04: 12: 00$ & 1.5 & $04: 13: 30$ & - & 00:03:00 & - \\
\hline 48 & 126 & 1 & $04: 13: 00$ & 437 & $04: 13: 30$ & 1.5 & $04: 15: 00$ & $00: 00: 30$ & - & 1 \\
\hline 49 & 347 & 3 & 04:16:00 & 753 & 04:16:00 & 2.5 & $04: 18: 30$ & - & 00:01:00 & - \\
\hline 50 & 646 & 6 & 04:22:00 & 663 & $04: 22: 00$ & 2.5 & $04: 24: 30$ & - & $00: 03: 30$ & - \\
\hline & & 329 & & & & 86 & & 00:03:00 & 04:01:00 & 3 \\
\hline
\end{tabular}

1. Average Queue length $=3 / 50=0.06$

2. Average waiting time of a customer $=3 / 50=0.06$ minutes.

3. Average service time $=86 / 50=1.72$ minutes.

4. Time a customer spends in the system $=0.06+1.72=1.78$ minutes.

5. Average waiting time of a server $=241 / 50=4.82$ minutes.

6. Average arrival time $=329 / 50=6.6$ minutes. 


V. Numerical Study
\begin{tabular}{|l|c|c|c|c|}
\hline \multicolumn{1}{|c|}{ Categories } & F1 & F2 & F3 & F4 \\
\hline Arrival Time & 2.14 & 4.7 & 4.4 & 6.6 \\
\hline Customer Waiting Time & 0.85 & 0.26 & 0.27 & 0.06 \\
\hline Average Service Time & 1.54 & 1.64 & 1.75 & 1.72 \\
\hline Queue Length & 0.46 & 0.26 & 0.26 & 0.06 \\
\hline
\end{tabular}

A. Comparison of Arrival time with Customer waiting time
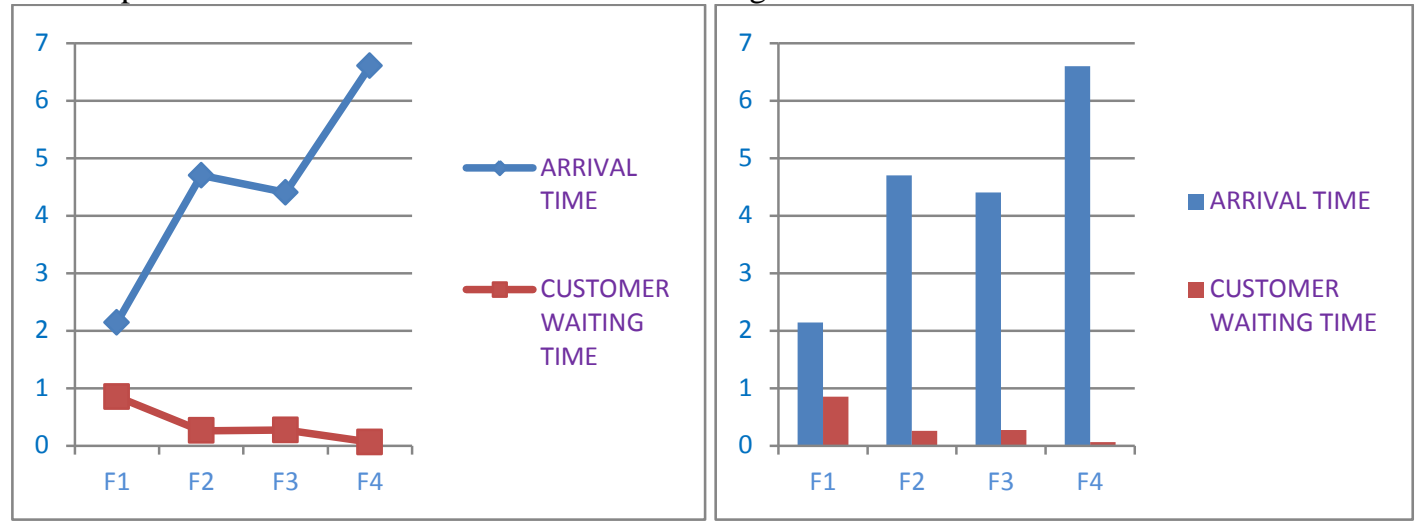

B. Comparison of Arrival time with Queue length
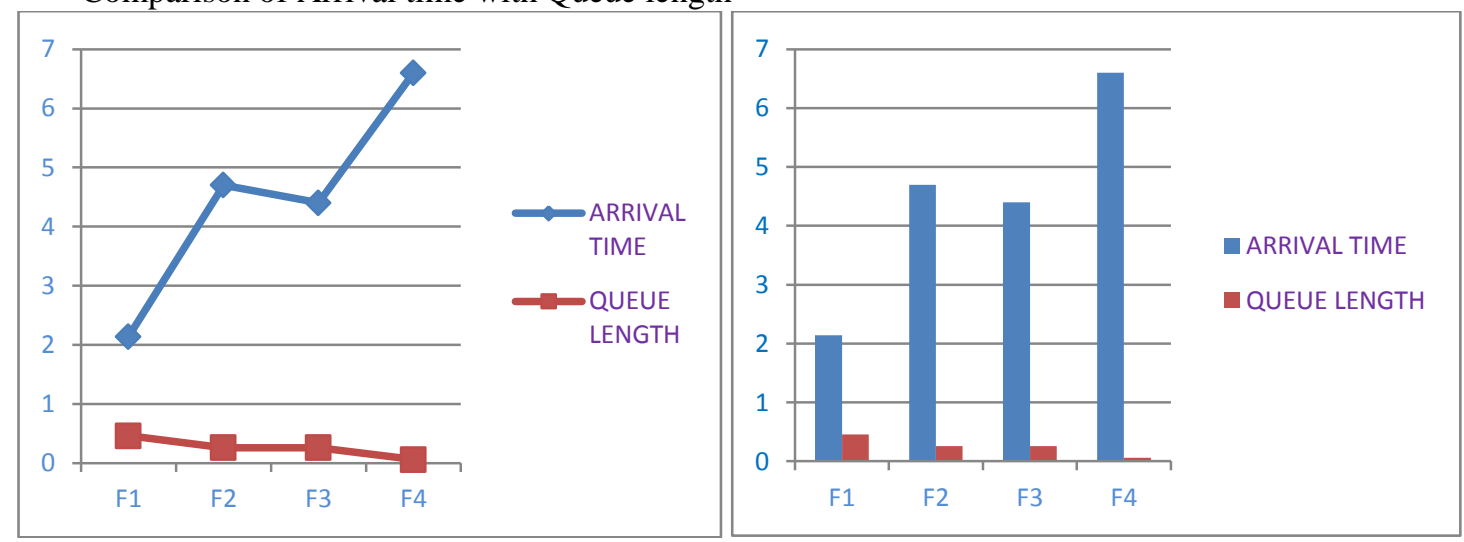

C. Comparison of Service time with Customer waiting time
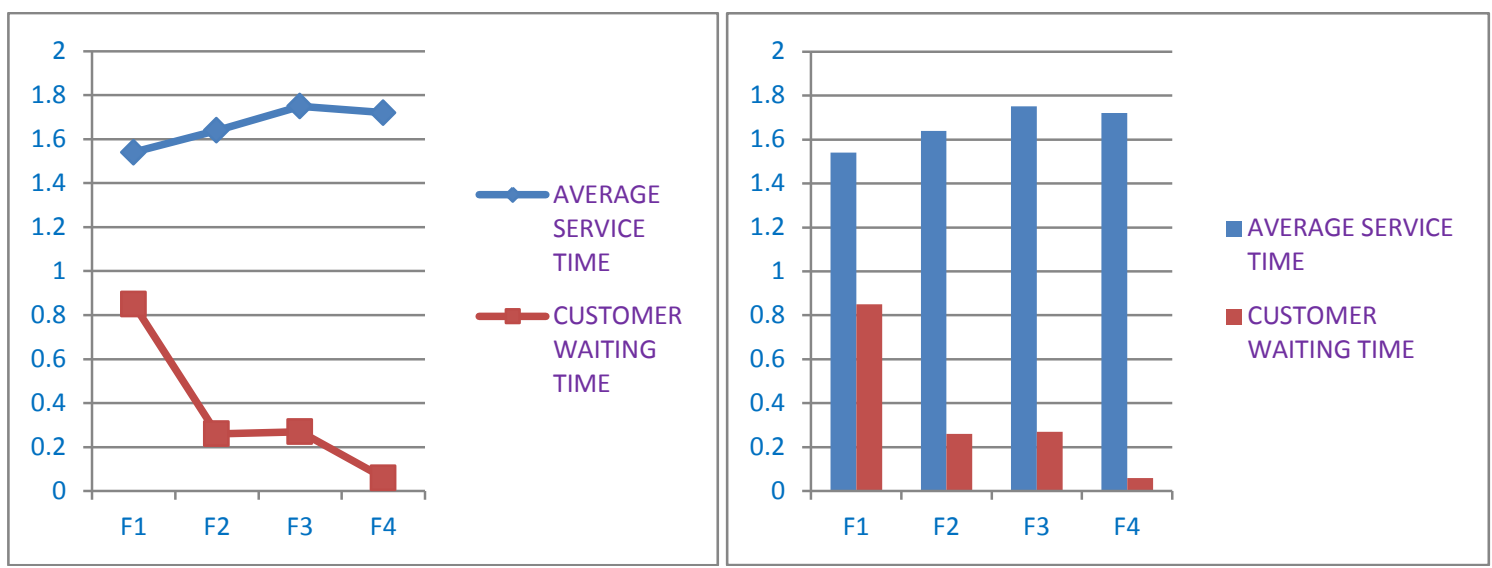
D. Comparison of Service time with Queue length

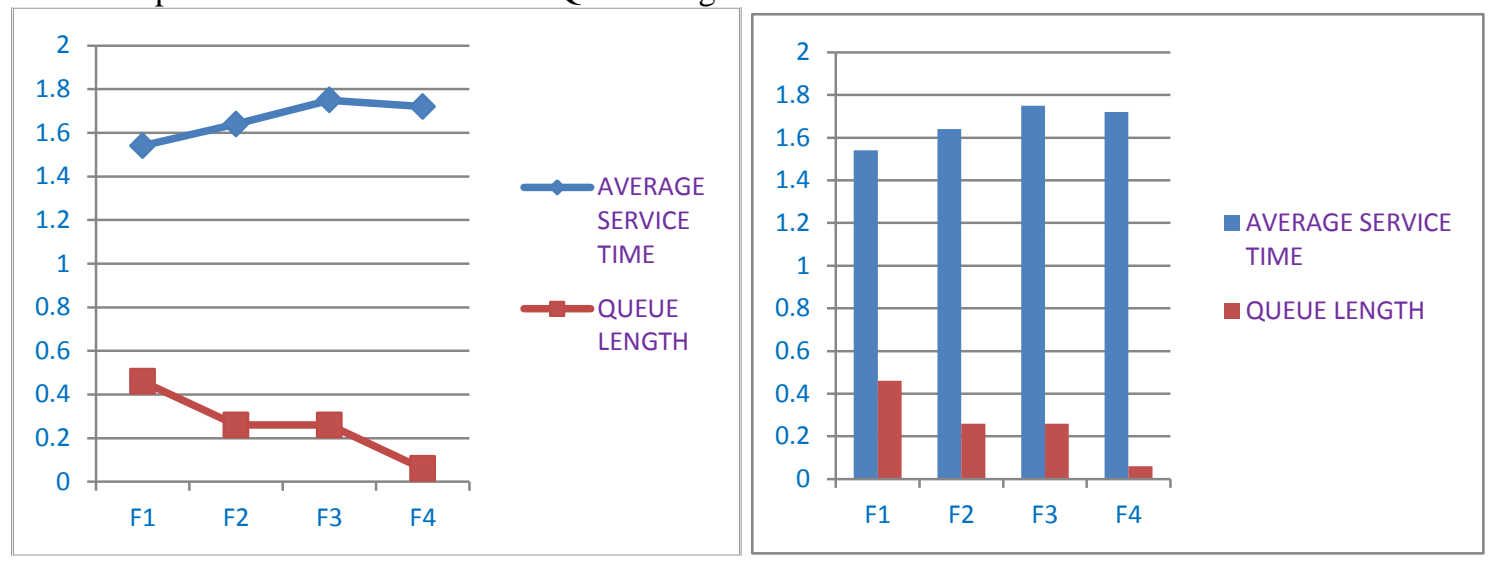

\section{Conclusion}

In above discussion we calculate the queue length, customer waiting time and average service times in toll gate. It is presented the basic ideas for simulation based approach on mathematical data. The proposed preliminary tollgate simulation table is hoped to be a starting point of my research. The numerical study shows the feasibility of the system. Further simulation studies are required to build a more comprehensive database in toll gate.

\section{References}

[1]. ^Benedettini,O., Tjahjono, B.(2008). "Towards an improved tool to facilitate simulation modeling of complex manufacturing systems". International Journal of Advanced Manufacturing Techology 43 (1/2): 191-9.doi:10.1007/s00170-008-1686-Z.

[2]. $\wedge \mathrm{a}$ b c Sundarapandian, V.(2009). "7.Queueing Theory". Probability, Statistics and Queueing Theory. PHI learning. ISBN 8120338448.

[3]. $\quad$ a b J.Banks, J Carson, B. Nelson, D.Nicol (2001). Discrete-Event System Simulation. Prentice Hall.p.3.ISBN 0-13-088702-1.

[4]. ^Also spelled queueing.askoxford.com. Retrived 2009-4-29.

[5]. ^Sokolowski, J.A., Banks, C.M (2009). Principles of Modeling and Simulation. Hoboken,NJ:Wiley.p.6.ISBN 978-0-470-28943-3.

[6]. Abbas-Turki, A., O.Grunder and A. Elmoudni. 2001. "Simulation and optimization of the public transportation connection system", In Proceedings of the $13^{\text {th }}$ European Simulation Symposium, October 18-20, Marseille, France,pp.435-439.

[7]. Biancadi, A., R. De Lotto and A. Ferrara. 2000. "A multimodal transport simulation tool to solve urban location problems", Proceedings of the $12^{\text {th }}$ European Simulation Sumposium, September 28-30, Humburg, Germany, pp.437-442.

[8]. Erlang, A.K, The theory of probabilities of telephone conversations, Nyt Jindsskriff Mathematic, B 20, 33-39(1909).

[9]. Fernandes, R.J. and S. Bampi. 1998. "A Software environment to integrate urban traffic simulation tasks", Proceedings of the 10" European Simulation Symposium, October 26-28, Nottingham, United Kingdom, pp.371-377.

[10]. Gale,C. and M.J.Oliveron and G.Silvan. 2002. "Simulation tool for managing a non-automated distribution warehouse", Proceedings of the $14^{\text {th }}$ European Simulation Symposium, October 23-26, Dresden, Germany,pp.266-270.

[11]. Ito,T.2004. "Simulation-based analysis of traffic jams at toll plaza with ETC gates", Proceedings of the Japan/USA Symposium on Fexible Automation, Denver, CO, July 19-21.

[12]. Ito, T. and T.Hiramoto. 2004. "Process simulation model towards analysis of traffic jams around toll gates", Information Technology Letters, Forum on Information Technology 2004, LO-002.

[13]. Ito,T. 2005. “ Process simulation approach to design and evaluation of toll plaza with ETC gates" International journal of simulation, Vol.6,No.5.

[14]. Lucjan,G.and O.Jozef. 1999. "Modeling of public transport commuters flow at urban interchange centers". Proceedings of the $11^{\text {th }}$ European Simulation Symposium, October 26-28, Erlanger, Germany,pp.217-219. 